\title{
A U.S. Geological Survey Round Robin for Microanalytical Techniques
}

\author{
H.A. Lowers, A.E. Koenig, S.A. Wilson, D.T. Adams, and G.P. Meeker \\ U.S. Geological Survey, MS 973, Denver, CO 80225
}

Many round robins and interlaboratory comparisons are conducted to test instrument and user proficiencies. The focus is typically on a single technique or single application such as glass reference materials for electron probe microanalysis (EPMA) [1,2,3,4,5] and glass and pressed powder pellet reference materials or candidate materials for laser ablation inductively coupled plasma mass spectroscopy (LA-ICP-MS) [5]. Most investigators, however, employ multiple microanalytical techniques such as EPMA, LA-ICP-MS, and secondary ion mass spectrometry (SIMS) to answer questions from major to trace element chemistry and isotopic composition. Difficulties may arise when comparing data obtained from various techniques or when using one set of data for internal calibration for another technique. Therefore, a two part round robin has been developed for the EPMA, SEM/EDS, LA-ICP-MS, SIMS, and atom probe communities to analyze the same material and initiate communication across instrument laboratories for methods and calibration. Basaltic glass materials have been chosen as the material for both parts of the round robin because they are easily doped with elements of interest, the method to produce the glass is well documented, and the glass can be used by all techniques. The glass will be spiked with elements that bridge the analytical strengths of the above listed techniques.

For the first part of the round robin, participants will follow explicit instructions regarding operating conditions and instrument parameters as well as utilizing provided reference materials. This part is intended to test the participants' instrument performance. For EPMA and EDS participants, the provided reference materials will consist of natural minerals and a previously characterized glass to be analyzed as an unknown. Analyzing the same materials will eliminate uncertainty due to inhomogeneity of the calibration materials. Standard blocks for LA-ICP-MS and SIMS participants will consist of NIST glasses, USGS glasses, and a previously characterized glass to be analyzed as an unknown. Each LA-ICP-MS and SIMS lab will receive their own standard block. EPMA and EDS participants will report k-ratios and concentrations. The reporting of k-ratios eliminates discrepancies which may arise from the use of different matrix correction algorithms (ie ZAF vs. PRZ). LA-ICP-MS and SIMS participants will report concentrations.

For the second part of the round robin, participants will receive a loose piece of a glass material to mount and analyze per their own protocols. This part of the round robin is to assess the laboratories' proficiency using their own in house reference materials and operating procedures.

The results of the two part round robin will be thoroughly discussed at a microanalytical reference materials topical conference in May of 2012. As part of the topical conference, not only will the compiled results, but also the observed challenges and suggestions for improvement of analyses and interlaboratory studies will be discussed. For their time and effort the laboratories will have a new reference material of which the certified values, or at the least, the consensus values from the round robin participants.

[1] E. Jarosewich et al., Smithsonian Cont. Earth Sci. 22 (1979) 53.

[2] J. Matousek et al., Pure \& Appl. Chem. 62 (1990) 1187.

[3] J. B. Hunt and P. G. Hill, Quaternary International, 34-36 (1996) 229.

[4] G. P. Meeker et al., Microsc. Microanal. 4 (suppl. 2) (1998) 240.

[5] P. Potts et al., Geostandards Newsletter 26 (2002) 197. 